\title{
3D Reconstruction of Zucchini- and Tobacco Yellow Mosaic Virus Induced Ultrastructural Changes in Plants
}

\author{
Bernd Zechmann ${ }^{1}$ and Günther Zellnig ${ }^{2}$ \\ 1. Baylor University, Center for Microscopy and Imaging, Waco, TX, USA. \\ 2. Karl-Franzens University of Graz, Institute of Plant Sciences, Graz, Austria.
}

Zucchini- and Tobacco Yellow Mosaic (ZYMV and TMV, respectively) are the most destructive and widespread virus diseases on Cucurbits and Tobacco worldwide. Even though they belong to different genera (Potyvirus and Tobamovirus, respectively) they induce similar symptoms on the plants such as stunting, yellowing and deformation of the leaves. In advanced stages of the infection, leaves develop yellow mosaic and often show dark green blisters [1,2]. Transmission electron microscopy (TEM) has been intensively applied to detect and identify ZYMV and TMV in the sap of plants, to characterize ultrastructural changes, and to rapidly diagnose these diseases in less than half a day $[3,4]$. These studies revealed that ZYMV induces the formation of cylindrical inclusions (CI) in the cytosol, while TMV simply accumulates in the cytosol in parallel form [3]. All of these studies focused on the 2 dimensional characterization of ZYMV and TMV diseases and therefore very little is known about how these changes affect the 3 dimensional ultrastructure of plant cells. In this study serial sections (section thickness was $80 \mathrm{~nm}$ ) of ZYMV and TMV infected leaves were 3D reconstructed by computer supported TEM in order to investigate 3D ultrastructural changes induced by ZYMV and TMV in leaves of Cucurbita pepo L. and Nicotiana tabacum L., respectively.

Results revealed that ZYMV induced the formation of cylindrical inclusions (CIs) which formed long tubular scroll like structures which had a preferred orientation throughout the cytosol (Figure 1). The average length of these CIs was determined at $3 \mu \mathrm{m}( \pm 0.2 \mu \mathrm{m})$, while the average width was found to be $120 \mathrm{~nm}( \pm 5 \mathrm{~nm})$. These sizes differed significantly from previous studies where a length and width of up to $20.1 \mu \mathrm{m}$ and $3.7 \mu \mathrm{m}$ were determined by confocal laser scanning microscopy (CLSM) [5]. These differences can be explained by the fact that the measurements in the latter studies reflected the fluorescence of the dyes bound to CIs rather than their exact size and that the width of CIs found in this study by TEM was below the resolution limit of CLSM. Additionally, we have noticed in this study that the beginning and end of CIs were often very closely associated with each other (below $200 \mathrm{~nm}$ ) making it impossible to differentiate individual CIs by CLSM. 3D reconstruction of TMV infected leaf cells revealed that virions accumulated into large spherical viral inclusion bodies throughout the cytosol that either occurred as single structures or were associated with each other or with other cell organelles (Figure 1). These bodies covered around $13 \%$ of the cytosol and virions accumulated inside these bodies in parallel form.

Summing up, the results presented in this study revealed 3D ultrastructural changes in ZYMV and TMV infected plants. ZYMV induced long tubular structures throughout the cytosol which are most probably involved in cell to cell movement through plasmodesmata. TMV induced spherical shaped inclusion bodies which contained virions aligned in parallel form. 
References:

[1] B Zechmann et al., Arch. Virol. 148 (2003), p. 1119.

[2] K Höller et al., Mol. Plant Microbe Interact. 23 (2010), p. 1448.

[3] B Zechmann and G Zellnig, J. Virol. Meth. 162 (2009), p. 163.

[4] G Zellnig et al., Microscopy 62 (2013), p. 547.

[5] TM Lim et al., J. Virol. Meth. 60 (1996), p. 139.
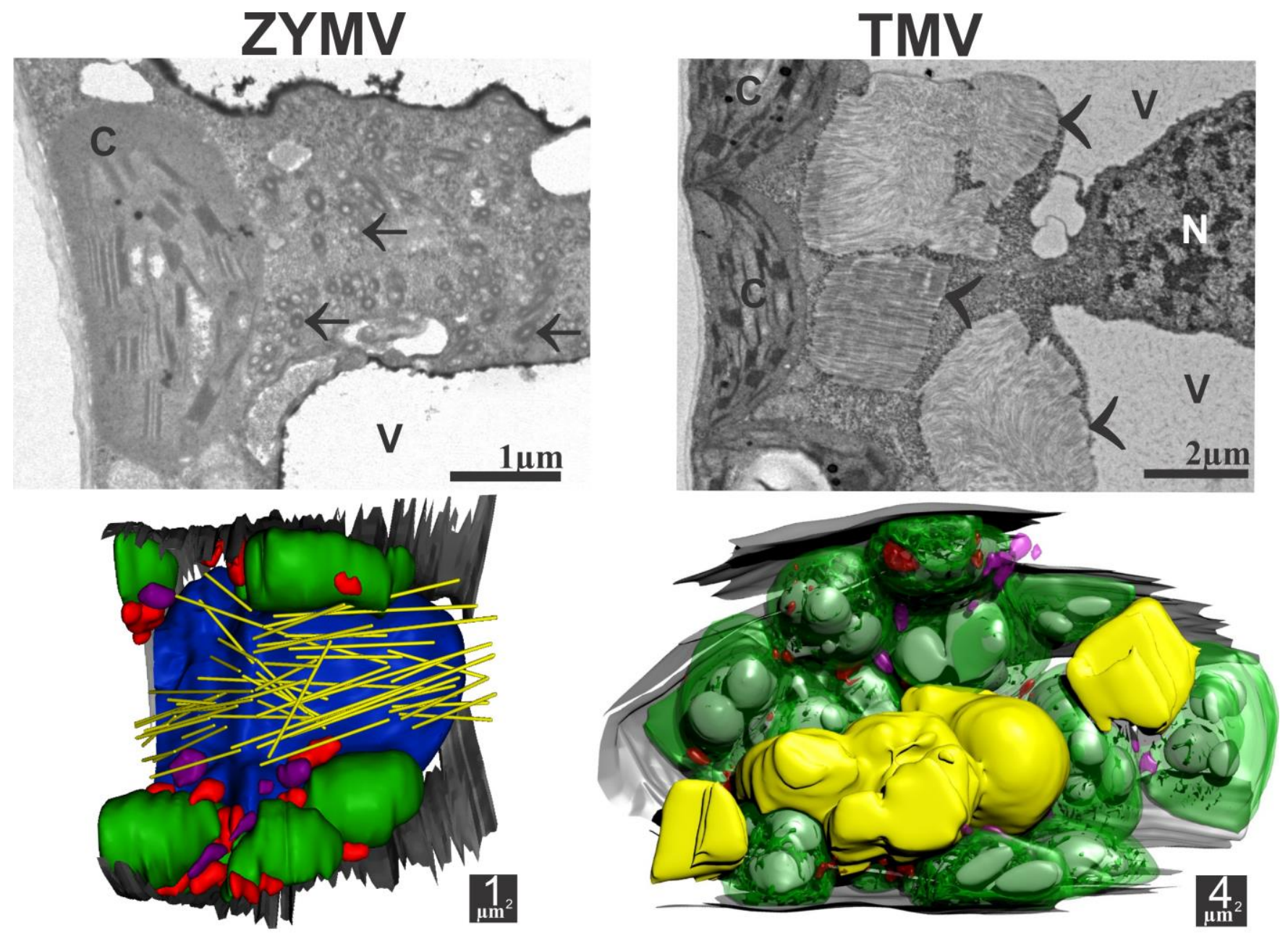

Figure 1. ZYMV and TMV-induced 2 and 3 dimensional changes in leaf cells of Cucurbita pepo and Nicotiana tabacum, respectively. ZYMV (left column) induced scroll like structures (arrows) which were organized in the form of long tubular cylinders (yellow structures) throughout the cytosol. TMV (right column) accumulated in parallel form within the cytosol of infected leaves (arrowheads) which formed spherical inclusion bodies (yellow bodies) inside the cytosol. $\mathrm{C}=$ chloroplasts, $\mathrm{N}=$ nucleus, $\mathrm{V}=$ vacuoles. Blue=vacuoles, gray=cell walls, green=chloroplasts, purple=peroxisomes, red=mitochondria. 